\section{Autoavaliação do estado de saúde e fatores associados: um estudo em trabalhadores bancários}

\author{
Self-assessment of health status and associated \\ factors: a study in bank workers
}

\author{
Autoevaluación del estado de salud y factores \\ asociados: un estudio en empleados del banco
}

\begin{abstract}
1 Universidade Federal do Espírito Santo, Vitória, Brasil.

Correspondência

G. B. Petarli

Universidade Federal do Espírito Santo.

Av. Marechal Campos 1468 Vitória, ES 29040-090, Brasil. glenda.petarli@gmail.com
\end{abstract}

\begin{abstract}
The aim of this study was to determine how bank employees assess their health status and risk factors associated with this indicator in this population. This is a cross-sectional study involving 525 workers of a banking system in the State of Espirito Santo, Brazil. The magnitude of the associations was assessed using logistic regression hierquizada in levels. It was found that $17 \%$ ( $n=87$ ) of bank self-rated their health status as fair or poor. Were associated with worse self-assessed health of the low socioeconomic level (OR=1.80; 95\%CI: 1.06-3.05), the sedentary lifestyle $(O R=2.64 ; 95 \% C I: 1.42-4.89)$, the excess weight $(O R=3.18 ; 95 \% C I$ : 1.79-5.65), low social support (OR = 3.71; 95\%CI: 2.10-6.58), and the presence of chronic diseases $(O R=5,49 ; 95 \% C I$ : 2.46-12.27). It is concluded that, compared with other locations, there was a significant number of banking that self-rated their health status as fair or poor, and that the presence of chronic diseases was presented as the factor with the greatest impact on how the individual evaluates their own health.
\end{abstract}

Psychological Stress; Occupational Health; Self-Assessment
Glenda Blaser Petarli 1

Luciane Bresciani Salaroli 1

Nazaré Souza Bissoli 1

Eliana Zandonade 1

\section{Resumo}

O objetivo deste trabalho foi verificar como trabalhadores bancários avaliam seu estado de saúde e os principais fatores associados a esse indicador nessa população. Trata-se de um estudo transversal com 525 funcionários de uma rede bancária do Estado do Espírito Santo, Brasil. A magnitude das associações foi avaliada através de regressão logística hierarquizada em níveis. Verificou-se que $17 \%(n=87)$ dos bancários autoavaliaram seu estado de saúde como regular ou ruim. Estiveram associados à pior autoavaliação de saúde o reduzido nível socioeconômico $(O R=1,80 ;$ IC95\%: 1,06-3,05), o estilo de vida sedentário (OR = 2,64; IC95\%: 1,42-4,89), o excesso de peso $(O R=3,18$; IC95\%: 1,79-5,65), o baixo apoio social $(\mathrm{OR}=3,71$; IC95\%: 2, 10-6,58) e a presença de doenças crônicas $(O R=5,49$; IC95\%: 2,46-12,27). Concluiu-se que, comparado com outras localidades, houve um expressivo número de bancários que autoavaliaram seu estado de saúde como regular ou ruim, e que a presença de doenças crônicas apresentou-se como o fator de maior impacto sobre a forma como o indivíduo avalia sua própria saúde.

Estresse Psicológico; Saúde do Trabalhador; Autoavaliação 


\section{Introdução}

Nas últimas décadas, a autoavaliação do estado de saúde tem sido um indicador amplamente utilizado em levantamentos epidemiológicos 1 . Esse indicador, determinado a partir de como o indivíduo avalia sua saúde em uma escala de quatro ou cinco pontos 2 , tem poder preditivo sobre a mortalidade, a morbidade e a utilização de serviços de saúde, além de refletir uma avaliação global de doenças, sintomas, habilidades funcionais e bem-estar geral 3. Por apresentar confiabilidade e validade equivalentes a outras avaliações mais complexas da condição de saúde, a autoavaliação do estado de saúde deve ser considerada um complemento importante para medidas objetivas 4 .

Os fatores levados em consideração pelo indivíduo ao autoclassificar seu estado de saúde ainda não são totalmente compreendidos, mas parecem refletir uma percepção abrangente de saúde que inclui aspectos biológicos, psicológicos e sociais 5 , assim como fatores demográficos, culturais e ainda aqueles relacionados ao ambiente de vida e de trabalho 1,6 .

Tendo em vista a estreita relação entre a autoavaliação de saúde com a mortalidade e a morbidade 5 , uma melhor compreensão dos fatores relacionados a esse indicador pode servir de base para o desenvolvimento de ações preventivas, de modo a manter ou a melhorar a saúde das populações. Nesse contexto, deve-se também ter atenção à saúde da população trabalhadora, em especial de setores submetidos a intensas reestruturações produtivas potencialmente prejudiciais ao indivíduo, como no caso de trabalhadores do setor bancário 7 .

Com o crescimento das trocas internacionais e dos investimentos estrangeiros, verificou-se a internacionalização das atividades bancárias. Cresceu a concorrência, que induziu transformações na organização do trabalho e adoção de novas tecnologias 8 . Se, de um lado, essas transformações acarretaram aumento da produtividade e lucratividade do setor, por outro lado geraram redução de emprego, maior exigência por qualificação e cumprimento de metas, intensificação e sobrecarga de tarefas, bem como aumento de controle e pressão sobre os trabalhadores, o que repercutiu fortemente nas condições de vida dos bancários 9 .

Sendo assim, este trabalho teve como objetivo verificar o modo como funcionários de uma rede bancária da Grande Vitória, Espírito Santo, Brasil, avaliam seu estado de saúde e os principais fatores associados a esse indicador em tal população.

\section{Material e métodos}

O estudo constituiu-se de uma investigação observacional transversal e seus dados foram derivados de uma pesquisa, junto a bancários, sobre síndrome metabólica, resistência à insulina e fatores associados 10. A amostra foi composta de 525 funcionários de uma rede bancária da região da Grande Vitória, com idade entre 20 a 64 anos, de ambos os sexos, em plena atividade laboral. A coleta de dados ocorreu no período de agosto de 2008 a agosto de 2009.

Para o cálculo do tamanho amostral considerou-se como população do estudo todos os 1.410 bancários da instituição onde foi realizada a pesquisa; levou-se em conta prevalência de autoavaliação positiva de saúde esperada de aproximadamente $50 \%$ (utilizados para maximizar a amostra), nível de significância de 5\%, erro amostral de $6 \%$ e efeito do desenho igual a dois (efeito dos conglomerados das agências). O tamanho mínimo da amostra calculada foi de 450 funcionários. Como forma de compensar as possíveis perdas, foram analisados todos os 525 bancários sorteados aleatoriamente para o projeto original.

As variáveis independentes foram agrupadas em sociodemográficas, comportamentais (hábitos de vida) e antropométricas, caraterísticas de trabalho e condição de saúde, coletadas a partir de questionário estruturado, aplicado por entrevistadores treinados. Essas variáveis foram incluídas segundo o modelo teórico apresentado na Figura 1, em que os blocos 1 e 2 apresentam possíveis variáveis de confusão que foram avaliadas no modelo multivariado.

As variáveis sociodemográficas incluíram sexo, faixa etária, escolaridade, etnia, estado civil e classe socioeconômica. A classe socioeconômica foi determinada de acordo com o Critério de Classificação Econômica Brasil (Associação Brasileira de Empresas de Pesquisa. http:/ /www. abep.org/novo/Content.aspx?ContentID=302, acessado em 29/Abr/2014). e categorizada em "A + B" e "C + D + E". A faixa etária foi categorizada em "até 30 anos", "entre 30 e 50 anos" e "mais de 50 anos".

O grupo de variáveis comportamentais e antropométricas incluíram consumo de bebida alcoólica, tabagismo, nível de atividade física, índice de massa corporal (IMC) e circunferência da cintura (CC).

Em relação ao tabagismo foram considerados fumantes os indivíduos que faziam uso do tabaco independente da quantidade ou frequência de consumo, conforme o consenso de abordagem e tratamento do fumante 11. 
Modelo teórico hierarquizado das possíveis relações entre variáveis sociodemográficas, comportamentais, antropométricas caraterísticas de trabalho e condição de saúde e a autoavaliação de saúde de trabalhadores bancários. Vitória, Espírito Santo, Brasil, 2008-2009.

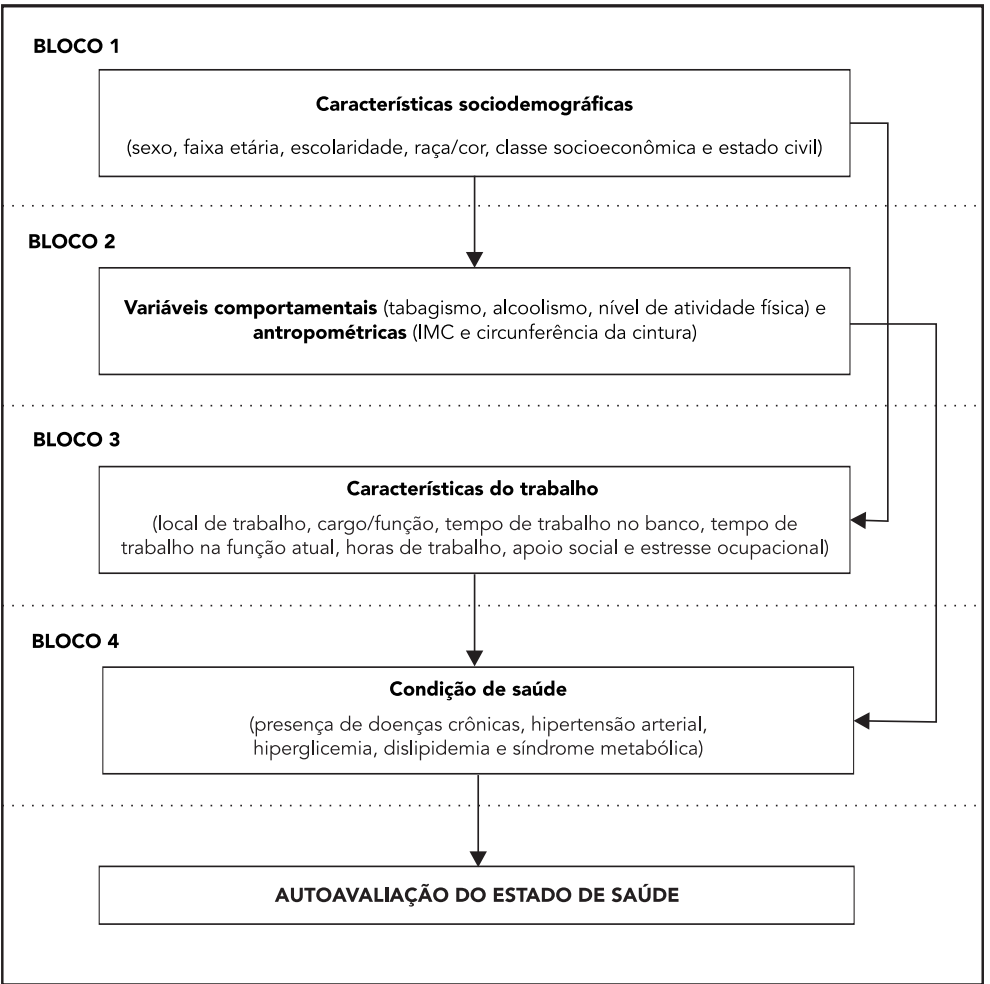

IMC: índice de massa corporal.

O nível de atividade física foi determinado pela aplicação da versão curta do Questionário Internacional de Atividade Física (IPAQ) validado para a população brasileira 12. Foram considerados indivíduos suficientemente ativos aqueles que relataram no mínimo 150 minutos de atividades com frequência $\geq 5$ dias na semana, levando-se em consideração apenas o somatório das sessões referentes a lazer e transporte, conforme recomendação atual, de modo a evitar a superestimação do nível de atividade física 13 .

O IMC foi categorizado de acordo com os pontos de corte da Organização Mundial da Saúde (OMS) 14 e reagrupados em eutrofia/baixo peso (IMC $\leq 24,9 \mathrm{~kg} / \mathrm{m}^{2}$ ) e sobrepeso/obesidade $\left(\right.$ IMC $\left.>24,9 \mathrm{~kg} / \mathrm{m}^{2}\right)$. A CC foi obtida através da medida, com fita métrica inextensível, da menor curvatura localizada entre o último arco costal e a crista ilíaca ${ }^{15}$. O ponto de corte adotado foi $\geq$ $94 \mathrm{~cm}$ para homens $\mathrm{e} \geq 80 \mathrm{~cm}$ para mulheres 14 .

O grupo de variáveis referentes às características do trabalho envolveu cargo/função, local de trabalho, tempo de trabalho no banco, tempo de trabalho na função atual, horas diárias de trabalho, apoio social e nível de estresse ocupacional. As variáveis relativas a tempo de trabalho no banco e tempo de trabalho na função atual foram categorizadas em "até cinco anos" e "mais de cinco anos", como forma de representar, respectivamente, os que trabalhavam há pouco tempo no banco ou na função atual e os que trabalhavam há mais tempo.

A variável cargo/função foi dicotomizada em "atendimento ao cliente" e "sem atendimento ao 
cliente". Os indivíduos classificados como "atendimento ao cliente" correspondiam àqueles que desempenhavam funções nas agências bancárias, tais como funções de caixa ou demais atribuições envolvidas com o atendimento direto ao público. Os "sem atendimento ao cliente" correspondiam àqueles indivíduos que desempenhavam cargos administrativos e/ou de gerência, realizando suas atividades sem contato direto com o público, em um prédio considerado a unidade administrativa, com estrutura física desvinculada das agências.

Para determinação do estresse ocupacional utilizou-se a versão reduzida e adaptada para o Brasil da Job Stress Scale desenvolvida para investigação do estresse ocupacional de acordo com o modelo demanda-controle 16. A partir da aplicação da escala, os escores obtidos na dimensão demanda e controle foram divididos em "alto" e "baixo" de acordo com as respectivas medianas. A partir da combinação entre os níveis de demanda e controle os indivíduos foram alocadas nos quatro quadrantes previstos no modelo: baixa exigência, trabalho ativo, trabalho passivo e alta exigência de forma a expressar as relações entre as demandas psicológicas e controle sobre o processo de trabalho ${ }^{17}$. O apoio social também foi determinado a partir da versão reduzida e adaptada para o Brasil da Job Stress Scale 16 e seus escores foram alocados em "alto" ou "baixo" de acordo com sua mediana.

O grupo de variáveis referentes à saúde envolveu a presença de doenças crônicas autorreferidas e alterações de saúde identificadas no estudo original. Em relação às doenças crônicas autorreferidas os indivíduos foram questionados se alguma vez receberam o diagnóstico de doenças como colesterol elevado, diabetes, úlcera, gastrite, hérnia de disco, lesão por esforço repetitivo (LER) e/ou artrose, sendo categorizadas em "nenhuma", “1 ou 2", "mais de 3 doenças".

Em relação às alterações diagnosticadas, foram coletados exames bioquímicos e variáveis hemodinâmicas para determinação de dislipidemia, pressão arterial elevada, síndrome metabólica e glicemia alterada. Maiores detalhes acerca da metodologia empregada no estudo original para coleta desses e dos demais dados estão publicados no estudo de Salaroli et al. 10 .

A classificação dos níveis pressóricos dos indivíduos foi feita com base nos critérios das $V I$ Diretrizes Brasileiras de Hipertensão 18. Neste estudo, reagrupou-se em: pressão arterial elevada (pressão arterial sistêmica - PAS $\geq 140 \mathrm{mmhg}$ e/ ou pressão arterial diastólica - $\mathrm{PAD} \geq 90 \mathrm{mmhg}$ ) e pressão arterial normal (abaixo destes valores). Deve-se destacar que também foram considerados hipertensos os indivíduos que relataram a utilização de medicamentos antihipertensivos.

Os níveis de glicemia foram classificados de acordo com as diretrizes da Sociedade Brasileira de Endocrinologia 19. Os indivíduos com glicemia menor ou igual a $100 \mathrm{mg} / \mathrm{dL}$ foram classificados na categoria de "glicemia normal" e os demais como "glicemia alterada".

Os níveis de LDL, colesterol total, triglicerídeos e HDL foram classificados de acordo com a V Diretriz Brasileira de Dislipidemias e Prevenção da Aterosclerose 20 para investigação de dislipidemia. O critério utilizado para o diagnóstico de síndrome metabólica foi do National Cholesterol Education Program - Adult Treatment Panel III dos Estados Unidos (NCEP-ATP-III) 21.

A variável dependente "autoavaliação do estado de saúde" foi analisada por meio da percepção do indivíduo sobre sua própria condição de saúde, com a pergunta: "De um modo geral, em comparação com pessoas de sua idade, como você considera o seu próprio estado de saúde?". As categorias de resposta "muito bom", "bom", "regular" e "ruim" foram reagrupadas em: "bom/ muito bom" e "regular/ruim".

Foram calculadas as frequências absolutas e relativas das variáveis independentes, segundo as categorias da autoavaliação do estado da saúde. Calculou-se o teste qui-quadrado para verificar associação entre elas; as variáveis com valor de p menor de $20 \%$ nesse teste foram incluídas na análise multivariada de regressão logística.

Para a análise de regressão logística foram realizados cinco modelos, em que as variáveis foram inseridas em blocos, conforme metodologia proposta por autores de estudo semelhante 22 . O Modelo 1 foi composto apenas pelas variáveis socioeconômicas; o Modelo 2, pelas variáveis socioeconômicas, comportamentais e antropométricas; o Modelo 3, composto por variáveis socioeconômicas, comportamentais, antropométricas, características do trabalho; o Modelo 4 e o Modelo 5 (final) foram constituídos por variáveis socioeconômicas, comportamentais, antropométricas, características do trabalho e condição de saúde.

Nos Modelos de 1 a 4, foi utilizado um método de inclusão das variáveis que determina a permanência de todas as variáveis no modelo, isto é, todas as variáveis são consideradas para o resultado dos odds ajustados. Para o modelo final, foi utilizado o método de inclusão das variáveis que as insere uma a uma, deixando no modelo ajustado apenas as variáveis estatisticamente significantes. Foram apresentados os odds ratio ajustados com os respectivos intervalos de 95\% de confiança. O nível de significância final adotado foi de $5 \%$. 
Este estudo foi aprovado pelo Comitê de Ética em Pesquisa do Centro de Ciências da Saúde da Universidade Federal do Espírito Santo (CAAE $n^{\circ}$ 03978612.5.0000.5060) como complementação do estudo original.

\section{Resultados}

Do total de 525 bancários do estudo original, foram elegíveis para esse estudo os dados de 520 indivíduos, por atenderem os critérios de inclusão. Desse total, $83 \%(n=433)$ dos indivíduos autoavaliaram seu estado de saúde como bom ou muito bom e $17 \%(n=87)$ autoavaliaram como regular ou ruim.

Na análise univariada, em relação às características sociodemográficas (Tabela 1), nenhuma variável apresentou-se significativamente associada à autoavaliação do estado de saúde.

Em relação às variáveis comportamentais e antropométricas (Tabela 2), foram associados à autoavaliação de saúde $(\mathrm{p}<0,05)$ o tabagismo ( $\mathrm{p}=0,020)$, o nível de atividade física $(\mathrm{p}=0,003)$, o IMC $(p=0,000)$ e a CC $(p=0,000)$. Entre os indivíduos que autoavaliaram negativamente seu estado de saúde, o maior percentual refere-se a pessoas que fumam e que possuem um nível insuficiente de atividade física, assim como a indivíduos que apresentam sobrepeso ou obesidade (IMC $>24,9 \mathrm{~kg} / \mathrm{m}^{2}$ ) e CC elevada.

A Tabela 3 apresenta a distribuição das variáveis relativas às características de trabalho segundo a autoavaliação de saúde. Nesse grupo, apenas o apoio social ( $\mathrm{p}=0,000)$ esteve associado ao desfecho investigado. Entre os que autoavaliaram seu estado de saúde como regular ou ruim, $69 \%$ apresentavam baixo apoio social.

Analisando-se as variáveis referentes à saúde (Tabela 4), verificou-se que esteve positivamente associada à autoavaliação de saúde a presença de doenças crônicas autorrelatadas $(p=0,000)$. Entre os que autorrelataram uma saúde regular ou ruim, mais de $80 \%$ correspondiam aos indi-

Tabela 1

Autoavaliação do estado de saúde, segundo características socioeconômicas e demográficas, em bancários da Grande Vitória, Espírito Santo, Brasil, 2008-2009

\begin{tabular}{|c|c|c|c|c|c|}
\hline \multirow[t]{3}{*}{ Variáveis } & \multicolumn{4}{|c|}{ Autoavaliação do estado de saúde } & \multirow[t]{3}{*}{ Valor de $p$ * } \\
\hline & \multicolumn{2}{|c|}{ Boa/Muito boa } & \multicolumn{2}{|c|}{ Regular/Ruim } & \\
\hline & $\mathbf{n}$ & $\%$ & $\mathbf{n}$ & $\%$ & \\
\hline \multicolumn{6}{|l|}{ Sexo } \\
\hline Feminino & 207 & 48 & 45 & 52 & 0,505 \\
\hline Masculino & 226 & 52 & 42 & 48 & \\
\hline \multicolumn{6}{|l|}{ Faixa etária (anos) } \\
\hline Até 30 & 81 & 19 & 14 & 16 & 0,268 \\
\hline Entre 30 e 50 & 260 & 60 & 60 & 69 & \\
\hline Mais de 50 & 92 & 21 & 13 & 15 & \\
\hline \multicolumn{6}{|l|}{ Escolaridade } \\
\hline 1ㅇ e 2o grau & 111 & 26 & 24 & 28 & 0,867 \\
\hline Universitário & 217 & 50 & 44 & 51 & \\
\hline Pós-graduado & 105 & 24 & 19 & 22 & \\
\hline \multicolumn{6}{|l|}{ Etnia } \\
\hline Branca & 257 & 59 & 45 & 52 & 0,188 \\
\hline Não branca & 176 & 41 & 42 & 48 & \\
\hline \multicolumn{6}{|l|}{ Estado civil } \\
\hline Casado/Companheiro & 275 & 64 & 59 & 68 & 0,761 \\
\hline Solteiro & 107 & 25 & 19 & 22 & \\
\hline Separado/Divorciado/Viúvo & 50 & 12 & 9 & 10 & \\
\hline \multicolumn{6}{|l|}{ Classe socioeconômica } \\
\hline$A+B$ & 247 & 57 & 41 & 47 & 0,089 \\
\hline$C+D+E$ & 186 & 43 & 46 & 53 & \\
\hline
\end{tabular}

* Teste qui-quadrado. 
Autoavaliação do estado de saúde, segundo características comportamentais e antropométricas, em bancários da Grande Vitória, Espírito Santo, Brasil, 2008-2009.

\begin{tabular}{|c|c|c|c|c|c|}
\hline \multirow[t]{3}{*}{ Variáveis } & \multicolumn{4}{|c|}{ Autoavaliação do estado de saúde } & \multirow[t]{3}{*}{ Valor de $p$ * } \\
\hline & \multicolumn{2}{|c|}{ Boa/Muito boa } & \multicolumn{2}{|c|}{ Regular/Ruim } & \\
\hline & $\mathbf{n}$ & $\%$ & $\mathbf{n}$ & $\%$ & \\
\hline \multicolumn{6}{|l|}{ Ingere bebida alcoólica } \\
\hline Sim & 274 & 63 & 52 & 60 & 0,701 \\
\hline Não & 130 & 30 & 30 & 34 & \\
\hline Bebi, mas parei & 29 & 7 & 5 & 6 & \\
\hline \multicolumn{6}{|l|}{ Fuma } \\
\hline Sim, regularmente & 34 & 8 & 15 & 17 & $0,020 * \star$ \\
\hline Não & 293 & 68 & 51 & 59 & \\
\hline Não, fumei, mas parei & 106 & 24 & 21 & 23 & \\
\hline \multicolumn{6}{|l|}{ Nível de atividade física } \\
\hline Suficientemente ativo & 161 & 37 & 18 & 21 & 0,003 ** \\
\hline Insuficientemente ativo & 272 & 63 & 69 & 79 & \\
\hline \multicolumn{6}{|l|}{$\mathrm{IMC}\left(\mathrm{kg} / \mathrm{m}^{2}\right)$} \\
\hline$\leq 24,9$ & 212 & 49 & 24 & 28 & 0,000 ** \\
\hline$>24,9$ & 221 & 51 & 63 & 72 & \\
\hline \multicolumn{6}{|l|}{ Circunferência da cintura } \\
\hline Normal & 224 & 52 & 26 & 30 & 0,000 ** \\
\hline Elevada & 208 & 48 & 61 & 70 & \\
\hline
\end{tabular}

víduos que apresentavam uma ou mais doenças crônicas autorreferidas.

A Tabela 5 apresenta os modelos com progressivos ajustes para as variáveis com valor de p menor de $20 \%$ na analise univariada. Após ajustes para as variáveis de todos os domínios, verificou-se que permaneceram associados à autoavaliação de saúde a classe socioeconômica, o nível de atividade física, o IMC, o apoio social e a presença de doenças crônicas.

Verificou-se que pertencer a classes socioeconômicas mais baixas aumentou o risco de os indivíduos autoavaliarem negativamente a própria saúde $(\mathrm{OR}=1,80$; IC95\%: 1,06-3,05), assim como ser insuficientemente ativo $(\mathrm{OR}=2,64$; IC95\%: 1,42-4,89) e apresentar excesso de peso $(\mathrm{OR}=3,18$; IC95: 1,79-5,65). No entanto, as variáveis que mais influenciaram na autoavaliação de saúde foi o reduzido apoio social, que elevou em mais de três vezes a chance de os indivíduos autoperceberem como regular ou ruim seu estado de saúde, e a presença de doenças crônicas. Os indivíduos com três ou mais doenças crônicas apresentaram maiores chances de pior autoa- valiação (OR = 5,49; IC95\%: 2,46-12,27), quando comparados aos indivíduos que relataram uma ou duas (OR = 3,12; IC95\%: 1,60-6,09).

\section{Discussão}

Nesse estudo, o número de indivíduos que autoavaliaram a saúde como regular ou ruim (17\%) foi aproximadamente duas vezes maior do que o observado em estudo semelhante envolvendo bancários de todas as capitais brasileiras 22 e similar ao percentual encontrado em trabalhadores industriais no Sul do Brasil 23. Entretanto, essa prevalência foi menor do que as encontradas em outros estudos nacionais, como no estudo Fatores de Risco e Proteção para Doenças Crônicas por Inquérito Telefônico (VIGITEL) 24 e a Pesquisa Nacional por Amostra de Domicílios (PNAD) 25, que verificaram autoavaliação negativa de saúde em $35,3 \%$ e $25,61 \%$ da população, respectivamente. Cabe ressaltar, no entanto que esses estudos, em especial o VIGITEL 24, apresentam diferenças metodológicas que podem comprometer a com- 
Autoavaliação do estado de saúde, segundo características do trabalho, em bancários da Grande Vitória, Espírito Santo, Brasil, 2008-2009.

\begin{tabular}{|c|c|c|c|c|c|}
\hline \multirow[t]{3}{*}{ Variáveis } & \multicolumn{4}{|c|}{ Autoavaliação do estado de saúde } & \multirow[t]{3}{*}{ Valor de $p$ * } \\
\hline & \multicolumn{2}{|c|}{ Boa/Muito boa } & \multicolumn{2}{|c|}{ Regular/Ruim } & \\
\hline & $\mathbf{n}$ & $\%$ & n & $\%$ & \\
\hline \multicolumn{6}{|l|}{ Cargo/Função } \\
\hline Sem atendimento ao cliente & 240 & 55 & 45 & 52 & 0,527 \\
\hline Atendimento ao cliente & 193 & 45 & 42 & 48 & \\
\hline \multicolumn{6}{|l|}{ Onde mora/trabalha } \\
\hline Sim & 283 & 65 & 59 & 68 & 0,659 \\
\hline Não & 150 & 35 & 28 & 32 & \\
\hline \multicolumn{6}{|l|}{ Tempo na função atual (anos) } \\
\hline Até 5 & 243 & 57 & 48 & 55 & 0,731 \\
\hline Mais de 5 & 182 & 43 & 39 & 45 & \\
\hline \multirow{2}{*}{\multicolumn{6}{|c|}{$\begin{array}{l}\text { Tempo de trabalho no banco } \\
\text { (anos) }\end{array}$}} \\
\hline & & & & & \\
\hline Até 5 & 116 & 27 & 21 & 24 & 0,584 \\
\hline Mais de 5 & 314 & 73 & 66 & 76 & \\
\hline \multicolumn{6}{|l|}{ Horas de trabalho diário } \\
\hline Até 6 & 178 & 41 & 34 & 39 & 0,725 \\
\hline Mais de 6 & 255 & 59 & 53 & 61 & \\
\hline \multicolumn{6}{|l|}{ Apoio social } \\
\hline Baixo apoio & 208 & 48 & 60 & 69 & $0,000 * *$ \\
\hline Alto apoio & 225 & 52 & 27 & 31 & \\
\hline \multicolumn{6}{|l|}{ Estresse ocupacional } \\
\hline Baixa exigência & 120 & 28 & 17 & 20 & 0,157 \\
\hline Trabalho ativo & 85 & 20 & 21 & 24 & \\
\hline Trabalho passivo & 152 & 35 & 27 & 31 & \\
\hline Alta exigência & 76 & 18 & 22 & 25 & \\
\hline
\end{tabular}

* Teste qui-quadrado;

$\star * \quad p<0,05$.

paração dos resultados, que deve, portanto, ser feita com cautela.

Considerando os resultados do modelo final ajustado, verificou-se que, em relação às variáveis sociodemográficas, diferentemente dos resultados de estudos nacionais 24,25 e internacionais 26,27 , esse estudo não encontrou diferenças na percepção de saúde com o aumento da idade, escolaridade ou sexo. No entanto, nível socioeconômico mais elevado mostrou-se positivamente associado à melhor autoavaliação de saúde, corroborando com a literatura disponível 25,27,28, inclusive com pesquisa envolvendo esse mesmo grupo de trabalhadores 22 .

A influência do nível socioeconômico na autoavaliação de saúde pode estar relacionada à aquisição de bens e produtos, incluindo medica- mentos e alimentos saudáveis; ao maior acesso a atividades de lazer e prática de exercícios físicos e à maior autonomia no trabalho que o dinheiro proporciona. Considerando que tais aspectos influenciam favoravelmente a saúde, indivíduos de maior nível socioeconômico tenderiam, portanto, a sentir-se mais saudáveis 28,29.

Apesar da influência positiva da atividade física sobre a saúde 30 , verificou-se que a maior parte dos bancários não destina tempo suficiente para a prática de atividade física, uma vez que, nessa pesquisa, mais da metade dos entrevistados foram considerados insuficientemente ativos. Os resultados também demonstraram que a inatividade física foi estatisticamente associada à pior autoavaliação de saúde. Tais resultados são condizentes com dados da literatura 22,23 e 
Autoavaliação do estado de saúde, segundo condição de saúde, em bancários da Grande Vitória, Espírito Santo, Brasil, 2008-2009.

\begin{tabular}{|c|c|c|c|c|c|}
\hline \multirow[t]{3}{*}{ Variáveis } & \multicolumn{4}{|c|}{ Autoavaliação do estado de saúde } & \multirow[t]{3}{*}{ Valor de $p$ * } \\
\hline & \multicolumn{2}{|c|}{ Boa/Muito boa } & \multicolumn{2}{|c|}{ Regular/Ruim } & \\
\hline & $\mathbf{n}$ & $\%$ & $\mathrm{n}$ & $\%$ & \\
\hline \multicolumn{6}{|c|}{ Doenças crônicas autorreferidas } \\
\hline Nenhuma & 183 & 43 & 16 & 19 & $0,000 * \star$ \\
\hline 1 ou 2 & 197 & 46 & 47 & 55 & \\
\hline 3 ou mais & 48 & 11 & 22 & 26 & \\
\hline \multicolumn{6}{|l|}{ Dislipidemia } \\
\hline Não & 197 & 47 & 30 & 36 & 0,055 \\
\hline Sim & 221 & 53 & 54 & 64 & \\
\hline \multicolumn{6}{|c|}{ Pressão arterial } \\
\hline Normal & 325 & 75 & 58 & 67 & 0,155 \\
\hline Elevada & 108 & 25 & 29 & 33 & \\
\hline \multicolumn{6}{|c|}{ Síndrome metabólica } \\
\hline Sim & 73 & 18 & 13 & 16 & 0,684 \\
\hline Não & 344 & 82 & 70 & 84 & \\
\hline \multicolumn{6}{|l|}{ Glicemia } \\
\hline Normal & 398 & 92 & 80 & 92 & 0,991 \\
\hline Alterada & 35 & 8 & 7 & 8 & \\
\hline
\end{tabular}

* Teste qui-quadrado;

** $p<0,05$.

reforçam as evidências de que os indivíduos que mantêm um estilo de vida mais saudável tendem a autoavaliar melhor seu estado de saúde quando comparados com aqueles que não o fazem 31 .

Apesar disso, essa investigação não encontrou significância estatística entre o tabagismo ou o consumo de álcool e a pior autoavaliação de saúde. Bombak 32 destaca que, apesar de alguns comportamentos serem considerados ostensivamente prejudiciais à saúde, para alguns indivíduos podem servir para promover prazer e alívio do estresse. Esse fato pode ter influenciado a forma como indivíduos que possuem o hábito de beber ou fumar autoavaliaram sua condição de saúde, comprometendo, consequentemente, a força de associação. A falta de associação com essas variáveis também foi evidenciada em outros estudos 1,33.

Corroborando com os resultados encontrados, está bem consolidado na literatura 34 que o excesso de peso aumenta as chances de pior autoavaliação de saúde. A obesidade, além de aumentar o risco para outras doenças crônicas, pode causar baixa autoestima, além de incapacidade funcional, problemas psicológicos e de interação social 35; essas condições podem ter impactado negativamente na forma como os bancários com excesso de peso perceberam sua saúde.

Com relação às características de trabalho, sabe-se que a exposição a condições de trabalhos adversas, extenuantes ou estressantes são fatores de risco para a saúde ${ }^{36}$. Apesar disso, de forma diferente do esperado, a exposição a trabalhos com maior risco de estresse ocupacional, caracterizado por alta demanda e baixo controle segundo o modelo demanda-controle, não esteve associada à pior autoavaliação de saúde quando se comparou à autoavaliação daqueles não expostos a tais tipos de atividades (trabalhos de baixa exigência).

Diferentemente desse estudo, Silva \& Barreto 22 , investigando bancários, verificaram uma chance duas vezes maior de autoavaliação negativa de saúde em indivíduos expostos ao maior risco de estresse comparado aos não expostos. Da mesma forma, estudos envolvendo outra categoria profissional 37,38 também verificaram associação positiva entre essas duas variáveis. Deve-se considerar que, devido à realidade multifacetada e subjetiva da percepção do estresse, outras formas de avaliá-lo, como abordagens 
Associação entre autoavaliação de saúde e variáveis sociodemográficas, comportamentais, antropométricas, características do trabalho e condição de saúde, segundo regressão logística multivariada, em bancários da Grande Vitória, Espírito Santo, Brasil, 2008-2009.

\begin{tabular}{|c|c|c|c|c|c|}
\hline Categoria & $\begin{array}{c}\text { Modelo } 1 \\
\text { OR (IC95\%) }\end{array}$ & $\begin{array}{c}\text { Modelo } 2 \\
\text { OR (IC95\%) }\end{array}$ & $\begin{array}{c}\text { Modelo } 3 \\
\text { OR (IC95\%) }\end{array}$ & $\begin{array}{c}\text { Modelo } 4 \\
\text { OR (IC95\%) }\end{array}$ & $\begin{array}{l}\text { Modelo } 5 \\
\text { OR (IC95\%) }\end{array}$ \\
\hline \multicolumn{6}{|l|}{ Etnia } \\
\hline Branca & 1,00 & 1,00 & 1,00 & 1,00 & \\
\hline Não branca & $1,32(0,83-2,11)$ & $1,26(0,77-2,06)$ & $1,26(0,76-2,09)$ & $1,10(0,96-1,88)$ & \\
\hline \multicolumn{6}{|c|}{ Classe socioeconômica } \\
\hline$C+D+E$ & $1,46(0,92-2,32)$ & $1,58(0,97-2,57)$ & $1,82(1,09-3,04)$ & $1,85(1,07-3,21)$ & $1,80(1,06-3,05)$ \\
\hline$A+B$ & 1,00 & 1,00 & 1,00 & 1,00 & 1,00 \\
\hline \multicolumn{6}{|l|}{ Fuma } \\
\hline Não & & 1,00 & 1,00 & 1,00 & \\
\hline Não, parei & & $1,00(0,56-1,80)$ & $1,01(0,55-1,84)$ & $0,89(0,47-1,69)$ & \\
\hline $\operatorname{Sim}$ & & $2,60(1,27-5,33)$ & $2,45(1,17-5,12)$ & $2,20(0,99-4,89)$ & \\
\hline \multicolumn{6}{|l|}{ Atividade física } \\
\hline Ativo & & 1,00 & 1,00 & 1,00 & 1,00 \\
\hline Inativo & & $2,44(1,37-4,35)$ & $2,64(1,46-4,78)$ & $2,89(1,53-5,46)$ & $2,64(1,42-4,89)$ \\
\hline \multicolumn{6}{|l|}{$\mathrm{IMC}\left(\mathrm{kg} / \mathrm{m}^{2}\right)$} \\
\hline$\leq 24,9$ & & 1,00 & 1,00 & 1,00 & 1,00 \\
\hline$>24,9$ & & $2,15(1,14-4,02)$ & $2,38(1,24-4,57)$ & $2,46(1,22-4,97)$ & $3,18(1,79-5,65)$ \\
\hline \multicolumn{6}{|c|}{ Circunferência da cintura } \\
\hline Normal & & 1,00 & 1,00 & 1,00 & \\
\hline Elevada & & $1,71(0,93-3,15)$ & $1,80(0,96-3,38)$ & $1,55(0,80-3,00)$ & \\
\hline \multicolumn{6}{|l|}{ Apoio social } \\
\hline Baixo apoio & & & $2,80(1,62-4,85)$ & $3,57(1,95-6,54)$ & $3,71(2,10-6,58)$ \\
\hline Alto apoio & & & 1,00 & 1,00 & 1,00 \\
\hline \multicolumn{6}{|l|}{ Estresse } \\
\hline Baixa exigência & & & 1,00 & 1,00 & \\
\hline Trabalho ativo & & & $1,54(0,72-3,27)$ & $1,20(0,54-2,68)$ & \\
\hline Trabalho passivo & & & $1,33(0,66-2,67)$ & $1,10(0,51-2,34)$ & \\
\hline Alta exigência & & & $1,79(0,84-3,80)$ & $1,42(0,63-3,16)$ & \\
\hline \multicolumn{6}{|l|}{ Doenças crônicas } \\
\hline Nenhuma & & & & 1,00 & 1,00 \\
\hline 1 ou 2 & & & & $2,96(1,50-5,84)$ & $3,12(1,60-6,09)$ \\
\hline 3 ou mais & & & & $5,03(2,19-11,53)$ & $5,49(2,46-12,27)$ \\
\hline \multicolumn{6}{|l|}{ Dislipidemia } \\
\hline Não & & & & 1,00 & \\
\hline $\operatorname{Sim}$ & & & & $1,34(0,75-2,40)$ & \\
\hline \multicolumn{6}{|l|}{ Pressão arterial } \\
\hline Normal & & & & 1,00 & \\
\hline Elevada & & & & $1,017(0,57-1,81)$ & \\
\hline
\end{tabular}

IC95\%: intervalo de 95\% de confiança; IMC: índice de massa corporal; OR: odds ratio.

Modelo 1: variáveis sociodemográficas; Modelo 2: variáveis sociodemográficas, comportamentais e antropométricas; Modelo 3: variáveis sociodemográficas, comportamentais, antropométricas, características do trabalho; Modelo 4: variáveis sociodemográficas, comportamentais, antropométricas, características do trabalho e condição de saúde; Modelo 5: modelo final, utilizando o método Forward LR de seleção de variáveis.

qualitativas ${ }^{39}$, poderiam ser de grande valia para ampliar a compreensão de seus efeitos sobre a saúde.
Apesar da ausência de associação com o estresse ocupacional, a variável relativa a apoio social, também obtida pela Job Stress Scale, man- 
teve-se fortemente associada à autoavaliação de saúde. No presente estudo, os bancários que relataram baixo apoio social apresentaram chance 3,71 vezes maior (IC95\%: 2,10-6,48) de perceberem sua saúde como ruim.

A explicação para tais resultados seria baseada no efeito protetor sobre a saúde que o apoio social exerceria, uma vez que a integração social, a confiança no grupo, o auxílio na execução de tarefas advindo de colegas e superiores, verificados em situações de elevado apoio social, poderiam atuar como protetores dos efeitos do desgaste no trabalho sobre a saúde ${ }^{40}$. Assim como em nosso estudo, outros autores 22,33,37 também demonstraram que os indivíduos com maiores índices de apoio social tendem a avaliar positivamente sua saúde.

Deve-se destacar, no entanto, que o fortalecimento do apoio social nas organizações de trabalho está cada vez mais prejudicado. O modelo atual de economia resulta, muitas vezes, em condições precárias de trabalho, desconfiança interpessoal e debilitação das relações sociais 41 . Além disso, condições frequentemente presentes na organização do trabalho bancário, como as avaliações individuais de desempenho, a gestão por metas e os programas de qualidade total são, segundo Dejours \& Bègue 42 , fatores que contribuem para a redução da solidariedade entre as pessoas, para a exacerbação do individualismo e para a competição desmesurada nos locais de trabalho, fragilizando ainda mais o apoio social entre os trabalhadores.

Apesar de o apoio social ter apresentado forte impacto na autoavaliação de saúde, a variável de maior influência foi a presença de doenças crônicas autorreferidas. No estudo de Barros et al. 24, a presença de doenças crônicas também permaneceu como a variável de maior impacto sobre o autoavaliação da saúde, aumentando em quase 12 vezes a chance de os indivíduos masculinos avaliarem negativamente sua saúde e, em sete vezes, em mulheres. Resultados semelhantes também foram encontrados por outros autores 22,43.

A relação entre autoavaliação de saúde e doenças crônicas pode ser explicada pelo fato de que, apesar de ter sido descrita na literatura a existência de muitos determinantes fisiológicos e psicossociais nessa autoavaliação, a dimensão física parece exercer maior influência sobre essa variável 44 . Portanto, considerando que os indivíduos com acúmulo de multimorbidades apresentam maiores chances de serem acometidos por dores, incapacidade, depressão, queixas somáticas, restrições na atividade física e na vida independente, eles tornam-se mais propensos a avaliar negativamente sua saúde 45 .
Cabe destacar o elevado número de bancários $314(61,2 \%)$ que autorreferiram possuir uma ou mais doenças crônicas. Dados da previdência social revelam que os principais motivos para concessão de auxílio-doença para trabalhadores do setor financeiro no ano de 2013 foram os relacionados a doenças osteomusculares, seguidos pelos transtornos mentais e comportamentais (Ministério da Previdência Social. Estatísticas: segurança e saúde ocupacional - tabelas. http:// www.previdencia.gov.br/estatisticas/menu-deapoio-estatisticas-seguranca-e-saude-ocupacio nal-tabelas/, acessado en 29/Abr/2014).

O comprometimento osteomuscular é muito frequente devido à natureza do trabalho bancário, com a manipulação de documentos, dinheiro e números, o que exige um esforço muscular estático o qual pode evoluir para inflamações nas estruturas músculo-esqueléticas 46 . Em função disso, algumas agências bancárias têm adotado medidas que visam auxiliar o trabalhador para que ele possa resistir por mais tempo na atividade, tais como ginásticas laborais, rodízios de funções e utilização de equipamentos e mobiliários ergonomicamente planejados 7 .

Os resultados desse estudo devem ser interpretados dentro do contexto de suas limitações. Entre elas, destaca-se o desenho metodológico que não permite fazer inferências causais ou temporais sobre associações encontradas. Além disso, algumas medidas foram baseadas em autorrelato e, portanto, podem estar sujeitas a viés de memória, suspeição diagnóstica e respostas socialmente desejáveis. Por fim, deve-se considerar que trabalhadores afastados das atividades laborais por motivos médicos não participaram da investigação, fato que pode ter subestimado a prevalência de autoavaliação negativa do estado de saúde 22 e interferido na magnitude das associações encontradas.

Como conclusão, pode-se afirmar que os resultados encontrados revelaram elevada prevalência de autoavaliação de saúde negativa entre os bancários investigados, quando comparados a bancários de outras localidades. Revelaram ainda que o fato de pertencer a classes socioeconômicas mais baixas, de apresentar estilo de vida sedentário, de estar acima do peso, de apresentar baixo apoio social no ambiente de trabalho e, principalmente, de possuir uma ou mais doenças crônicas impactou negativamente na forma como os bancários avaliaram seu próprio estado de saúde.

Considerando esses resultados, percebe-se que a maior parte dos fatores que influenciaram negativamente na autoavaliação de saúde dos bancários é passível de controle e orientação. Assim, torna-se necessário haver estratégias orga- 
nizacionais focadas tanto no indivíduo, voltadas para o estímulo da prática de atividade física e para o controle de peso, quanto na organização do trabalho, com vistas à integração no ambiente laboral, de modo a favorecer o apoio social e a melhoria constante nos cuidados à saúde desses trabalhadores, considerando-se a promoção, a prevenção e a intervenção como fatores fundamentais para a melhoria das condições de saúde e trabalho.

\section{Resumen}

El objetivo de este estudio fue investigar cómo los empleados del banco a evaluar su estado de salud y factores de riesgo asociados a este indicador en esta población. Se trata de un estudio transversal con 525 trabajadores de un sistema bancario en el estado de Espírito Santo, Brasil. La magnitud de las asociaciones se evaluó mediante regresión logística hierquizada en niveles. Se encontró que $17 \%(n=87)$ de los trabajadores del banco auto-calificaron su estado de salud como regular o mala. Se asociaron con la salud autopercibida peor del bajo nivel socio-económico $(O R=1,80$; IC95\%: 1,06-3,05), el sedentarismo (OR $=2,64 ;$ IC95\%: 1,42 4,89), el exceso de peso (OR $=3,18$; IC95\%: $1,79-5,65)$, el baja apoyo social (OR = 3,71; IC95\%: 2,10-6,58), y la presencia de enfermedades crónicas $(O R=5,49 ;$ IC95\%: $2,46-12,27)$. Se concluye que, en comparación con otros lugares, hubo un número significativo de la banca que la auto-calificaron su estado de salud como regular o mala, y que la presencia de enfermedades crónicas se presenta como el factor de mayor impacto en cómo los evalúa individuales su propia salud.

Estrés Psicológico; Salud Laboral;

Autoevaluación

\section{Colaboradores}

G. B. Petarli, L. B. Salaroli, N. S. Bissoli e E. Zandonade participaram da concepção do projeto, na análise e interpretação dos dados, na redação do artigo, na revisão crítica relevante do conteúdo intelectual e na aprovação final da versão a ser publicada.

\section{Agradecimentos}

Os autores gostariam de agradecer a todos os funcionários do banco que colaboraram para este estudo e à instituição financeira, que permitiu e financiou o desenvolvimento desta pesquisa. 


\section{Referências}

1. Garcia LP, Höfelmann DA, Facchini LA. Self-rated health and working conditions among workers from primary health care centers in Brazil. Cad Saúde Pública 2010; 26:971-80.

2. Wu S, Wang R, Zhao Y, Ma X, Wu M, Yan X, et al. The relationship between self-rated health and objective health status: a population-based study. BMC Public Health 2013; 13:320.

3. Quesnel-Vallée A. Self-rated health: caught in the crossfire of the quest for "true" health? Int J Epidemiol 2007; 36:1161-4.

4. Mood C. Life-style and self-rated global health in Sweden: a prospective analysis spanning three decades Prev Med 2013; 57:802-6.

5. Guimarães JMN, Chor D, Werneck GL, Carvalho MS, Coeli CM, Lopes CS, et al. Association between self-rated health and mortality: 10 years follow-up to the Pró-Saúde cohort study. BMC Public Health 2012; 12:676.

6. Kelleher CC, Friel S, Gabhainn SN, Tay JB. Sociodemographic predictors of self-rated health in the Republic of Ireland: findings from the National Survey on Lifestyle, Attitudes and Nutrition, SLAN. Soc Sci Med 2003; 57:477-86.

7. Murofuse NT, Marziale MHP. Mudanças no trabalho e na vida de bancários portadores de lesões por esforços repetitivos: LER. Rev Latinoam Enferm 2001; 9:19-25.

8. Larangeira SMG. Reestruturação produtiva no setor bancário: a realidade dos anos 90 . Educação \& Sociedade 1997; 18:110-38.

9. Silva JL, Navarro VL. Organização do trabalho e saúde de trabalhadores bancários. Rev Latinoam Enferm 2012; 20:226-34.

10. Salaroli LB, Saliba RAD, Zandonade E, Molina MC, Bissoli NS. Prevalence of metabolic syndrome and related factors in bank employees according to different defining criteria, Vitória/ES, Brazil. Clinics 2013; 68:69-74.

11. Instituto Nacional de Câncer. Abordagem e tratamento do fumante: consenso 2001. Rio de Janeiro: Instituto Nacional de Câncer; 2001.

12. Matsudo S, Araújo T, Matsudo V, Andrade D, Andrade E, Oliveira LC, et al. Questionário Internacional de Atividade Física (IPAQ): estudo de validade e reprodutibilidade no Brasil. Rev Bras Ativ Fís Saúde 2001; 6:5-18.

13. Hallal PC, Gomez LF, Parra DC, Lobelo F, Mosquera J, Florindo AA, et al. Lições aprendidas depois de 10 anos de uso do IPAQ no Brasil e Colômbia. J Phys Act Health 2010; 7:259-64.

14. World Health Organization. Obesity: preventing and managing the global epidemic. Geneva: World Health Organization; 1998. (WHO Technical Report Series, 894).

15. Duarte ACG. Avaliação nutricional: aspectos clínicos e laboratoriais. São Paulo: Editora Atheneu; 2007.

16. Alves MGM, Chor D, Faerstein E, Lopes CS, Werneck GL. Versão resumida "job stress scale”: adaptação para o português. Rev Saúde Pública 2004; 38:164-71.
17. Karasek RA. Job demands, job decision latitude, and mental strain: implications for redesingn. Adm Sci Q 1979; 24:285-308.

18. Brandão AA, Rodrigues CIS, Consolim-Colombo F, Plavnik FL, Malachias MVB, Kohlmann Junior O, et al. VI diretrizes brasileiras de hipertensão. Arq Bras Cardiol 2010; 95(1 Suppl 1):I-III.

19. Sociedade Brasileira de Endocrinologia e Metabologia. Diabetes mellitus: classificação e diagnóstico. Projeto Diretrizes. http://www.projetodiretri zes.org.br/4_volume/06-diabetes-c.pdf (acessado em 29/Abr/2014).

20. Xavier HT, Izar MC, Faria Neto JR, Assad MH, Rocha VZ, Sposito AC, et al. V diretriz brasileira de dislipidemias e prevenção da aterosclerose. Arq Bras Cardiol 2013; 101(4 Suppl 1):1-20.

21. Expert Panel on Detection, Evaluation, and Treatment of High Blood Cholesterol in Adults. Executive summary of the Third Report of the National Cholesterol Education Program (NCEP) Expert Panel on Detection, Evaluation, and Treatment of High Blood Cholesterol in Adults (Adult Treatment Panel III). JAMA 2001; 285:2486-97.

22. Silva LS, Barreto SM. Stressful working conditions and poor self-rated health among financial services employees. Rev Saúde Pública 2012; 46:407-16.

23. Höfelmann DA, Blank N. Auto-avaliação de saúde entre trabalhadores de uma indústria no sul do Brasil. Rev Saúde Pública 2007; 41:777-87.

24. Barros MBA, Zanchetta LM, Moura EC, Malta DC. Auto-avaliação da saúde e fatores associados, Brasil, 2006. Rev Saúde Pública 2009; 43:27-37.

25. Dachs JW, Santos APR. Auto-avaliação do estado de saúde no Brasil: análise dos dados da PNAD/2003. Ciênc Saúde Coletiva 2006; 11:887-94.

26. Shin HY, Shin MH, Rhee JA. Gender differences in the association between self-rated health and hypertension in a Korean adult population. BMC Public Health 2012; 12:135.

27. Alvarez-Galvez J, Rodero-Cosano ML, Motrico E, Salinas-Perez JA, Garcia-Alonso C, Salvador-Carulla L. The impact of socio-economic status on selfrated health: study of 29 countries using European Social Surveys (2002-2008). Int J Environ Res Public Health 2013; 10:747-61.

28. Peres MA, Masiero AV, Longo GZ, Rocha GC, Matos IB, Najnie K, et al. Auto-avaliação da saúde em adultos no Sul do Brasil. Rev Saúde Pública 2010; 44:901-11.

29. Popkin BM, Siega-Riz AM, Haines PS. A comparison of dietary trends among racial and socioeconomic groups in the United States. N Engl J Med 1996; 335:716-20.

30. World Health Organization. Global recommendations on physical activity for health. Geneva: World Health Organization; 2010.

31. Darviri C, Artemiadis AK, Tigani X, Alexopoulos EC. Lifestyle and self-rated health: a cross-sectional study of 3,601 citizens of Athens, Greece. BMC Public Health 2011; 11:619.

32. Bombak AE. Self-rated health and public health: a critical perspective. Front Public Health 2013; 1:15. 
33. Malinauskiene V, Leisyte P, Romualdas M, Kirtiklyte K. Associations between self-rated health and psychosocial conditions, lifestyle factors and health resources among hospital nurses in Lithuania. J Adv Nurs 2011; 67:2383-93

34. Prosper M, Moczulski VL, Qureshi A. Obesity as a predictor of self-rated health. Am J Health Behav 2009; 33:319-29.

35. Cabrera MAS, Jacob Filho W. Obesidade em idosos: prevalência, distribuição e associação com hábitos e comorbidades. Arq Bras Endocrinol Metab 2001; 45:494-501.

36. Hämmig O, Bauer GF. Work, work-life conflict and health in an industrial work environment. Occup Med (Lond) 2014; 64:34-8.

37. Griep RH, Rotenberg L, Landsbergis P, Vasconcellos-Silva PR. Uso combinado de modelos de estresse no trabalho e a saúde auto-referida na enfermagem. Rev Saúde Pública 2011; 45:145-52.

38. Theme Filha MM, Costa MAS, Guilam MCR. Estresse ocupacional e autoavaliação de saúde entre profissionais de enfermagem. Rev Latinoam Enferm 2013; 21:475-83

39. Halford C, Anderzén I, Arnetz B. Endocrine measures of stress and self-rated health: a longitudinal study. J Psychosom Res 2003; 55:317-20.

40. Johnson JV, Hall EM. Job strain, workplace social support and cardiovascular disease: a cross-sectional study of a random sample of the Swedish working population. Am J Public Health 1988; 78:1336-42.
41. Moyano Diaz E, Barría R. Suicidio y producto interno bruto (PIB) en Chile: hacia un modelo predictivo. Rev Latinoam Psicol 2006; 38:343-59.

42. Ferreira LL. Suicide et travail, que faire? [Resenha]. Rev Bras Saúde Ocup 2009; 34:184-5.

43. Ramkumar A, Quah JL, Wong T, Yeo LS, Nieh CC, Shankar A, et al. Self-rated health, associated factors and diseases: a community-based cross-sectional study of Singaporean adults aged 40 years and above. Ann Acad Med Singapore 2009; 38: 606-12.

44. Barros MBA. Auto-avaliação de saúde. In: Cesar CLG, Carandina L, Alves MCGP, Barros MBA, Goldbaum M, organizadores. Saúde e condição de vida em São Paulo. Inquérito multicêntrico de saúde no Estado de São Paulo - ISA-SP. São Paulo: Faculdade de Saúde Pública, Universidade de São Paulo; 2005. p. 173-82.

45. Nützel A, Dahlhaus A, Fuchs A, Gensichen J, König HH, Riedel-Heller S. Self-rated health in multimorbid older general practice patients: a crosssectional study in Germany. BMC Fam Pract 2014; $15: 1$.

46. Alves PHR. O psicossocial e a LER. In: Oliveira CR, Alves PHR, organizadores. Manual prático de LER: lesões por esforços repetitivos. 2a Ed. Belo Horizonte: Health; 1998. p. 33-51.

Recebido em 28/Mai/2014

Versão final reapresentada em 06/Nov/2014 Aprovado em 17/Nov/2014 